\title{
Direct Measurement of the Quantum Wavefunction using Weak Measurements in Orbital Angular Momentum
}

\author{
Mehul Malik, ${ }^{1}$ Mohammad Mirhosseini, ${ }^{1}$ Martin P. J. Lavery, ${ }^{2}$ Malcolm O'Sullivan, ${ }^{1}$ Miles J. \\ Padgett,$^{2}$ and Robert W. Boyd ${ }^{1,3}$ \\ ${ }^{1}$ The Institute of Optics, University of Rochester, Rochester, New York 14627 USA \\ ${ }^{2}$ School of Physics and Astronomy, University of Glasgow, Glasgow, United Kingdom \\ ${ }^{3}$ Department of Physics, University of Ottawa, Ottawa, ON K1N 6N5 Canada \\ memalik@optics.rochester.edu
}

\begin{abstract}
We propose a method that directly measures the photon wavefunction in the orbital angular momentum (OAM) basis by carrying out weak measurements in OAM and strong measurements in the conjugate basis of angular position.
\end{abstract}

(C)2011 Optical Society of America

OCIS codes: (270.0270) Quantum Optics; (000.2658) Fundamental Tests

In Lundeen et al.'s recent paper [1], the authors describe a novel way of directly measuring the complex wavefunction of a photon by performing a weak measurement, which does not disturb the state of the photon altogether. Using polarization as a measurement pointer, they perform a weak measurement in position (x) followed by a strong measurement in momentum (p), which lets them measure the real and imaginary part of the single-photon wavefunction. Their technique surpasses present tomographic methods of measuring the wavefunction in that it does not require any indirect computational reconstruction.

We propose an extension of this method to the orbital angular momentum (OAM) basis. We aim to weakly measure the orbital angular momentum $\left(A=\pi_{l}=|l\rangle\langle l|\right)$ of a photon and strongly measure its angular position $(\theta)$. In this case, the weak value will be

$$
\left\langle\pi_{l}\right\rangle_{w}=\frac{\langle\theta \mid l\rangle\langle l \mid \Psi\rangle}{\langle\theta \mid \Psi\rangle}=\frac{e^{i l \theta / h} \Psi(l)}{\Phi(\theta)}
$$

In the case when $\theta=\theta_{0}$, this simplifies to

$$
\left\langle\pi_{l}\right\rangle_{w}=a \Psi(l)
$$

where $a=1 / \Phi\left(\theta_{0}\right)$ is a constant. If we choose the strong measurement of $\theta_{0}$ appropriately, we see that the average result of the weak measurement of $\pi_{l}$ is proportional to the wavefunction of the particle in $l$. In keeping with the language used in [1], the observed position and momentum shifts of the measurement pointer are proportional to $\operatorname{Re} \Psi(l)$ and $\operatorname{Im} \Psi(l)$. By scanning through a discrete set of $l$, we can directly measure the complex wavefunction in the orbital angular momentum basis.

Carrying out a weak measurement of OAM does not seem like a trivial task. However, a solution is apparent in the method proposed in [2], where the authors describe a set of refractive elements that map a photon's orbital angular momentum to its position. Using this OAM sorter, we are able to carry out weak measurements in the orbital angular momentum basis, by mapping $l$ to discrete $x$ and then rotating the polarization of the discrete position that we want to weakly measure.

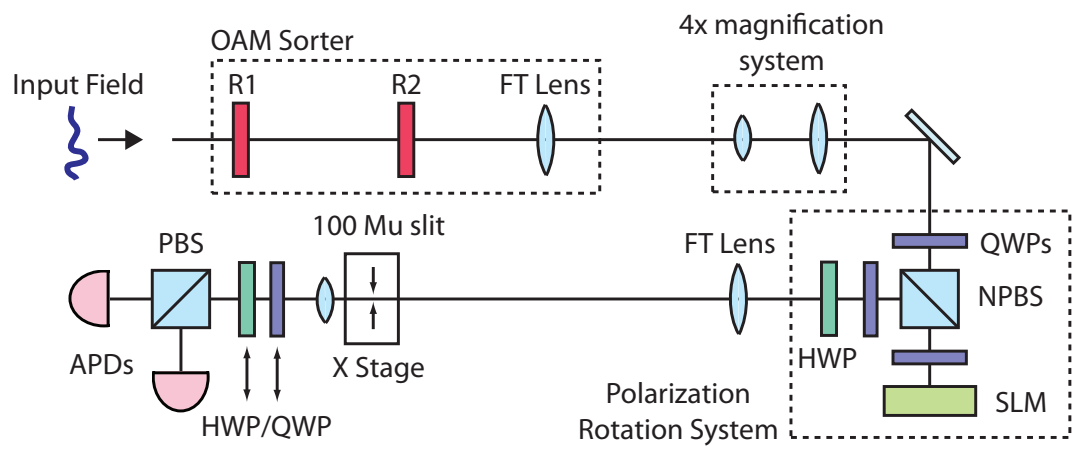

Figure 1: Schematic of our experimental setup. 
a)

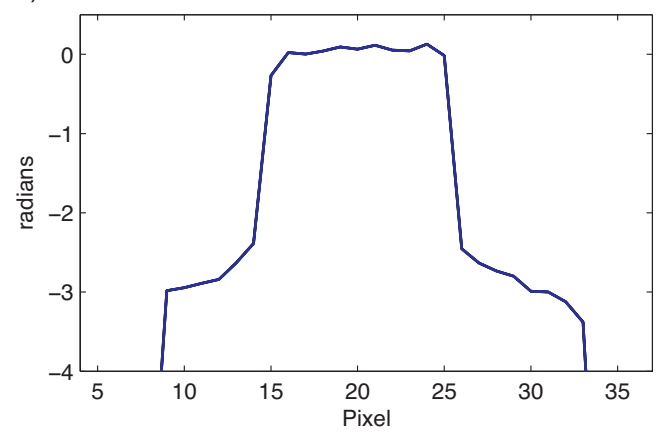

b)

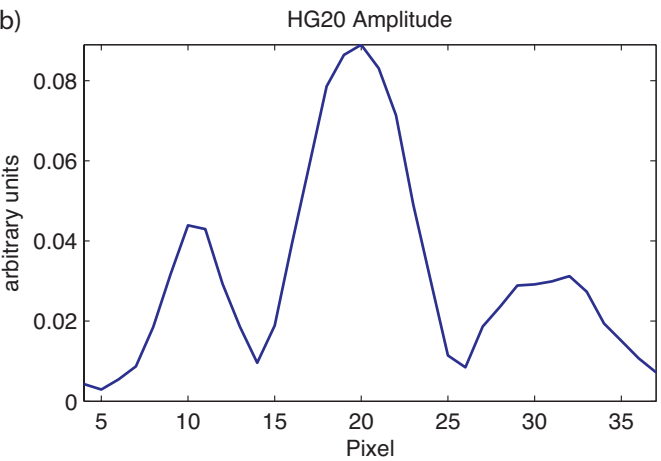

Figure 2: Preliminary results showing (a) the phase and (b) the amplitude of an HG20 beam as measured by our experimental setup shown in Figure 1. The phase of the two sidelobes relative to the center is measured to be 3.01 radians and 3.06 radians.

A key difference between our experimental implementation (shown in Fig. 1) and that of [1] is that we rotate the polarization of the field by using a phase-only spatial light modulator (SLM) in conjunction with three quarter-wave plates (QWP) and a half-wave plate (HWP). This is based on a method that is explained in [3], which allows for faster and finer controlled weak measurements. This is a stark improvement over the method of physically moving a waveplate sliver used in [1]. The strong measurement is carried out with a $100 \mu \mathrm{m}$ slit in the fourier plane of a lens after the SLM. However, since the sorter maps $l$ to $x$ and $\theta$ to $p$, we are strongly measuring in angular position $(\theta)$ in the fourier plane of the lens.

The rest of the analysis requires that we measure the $S_{2}$ and $S_{3}$ components of the stokes polarization vector, as explained in [1]. This is carried out with a HWP (for $S_{2}$ ) and a QWP (for $S_{3}$ ) and a polarizing beam splitter (PBS). The signal imbalance between the two output ports of the PBS is measured using photoncounting detectors (APDs). The measured stokes components allow us to calculate the complex weak value [1] for each value of OAM $(l)$, and its real and imaginary parts are used to calculate the amplitude and phase of the wavefunction as a function of $l$.

As a test of our experimental setup, we measured the phase and amplitude of an HG20 photon without the OAM sorter in place. Preliminary results are shown in Fig. 2. We have simulated the direct measurement of a complex OAM superposition of $l= \pm 1, \pm 3, \pm 5$ in Fig. 3. The input intensity distribution and the measured phase of the photon in the OAM basis is plotted. We expect to have experimental results to support these simulated results soon.

a)

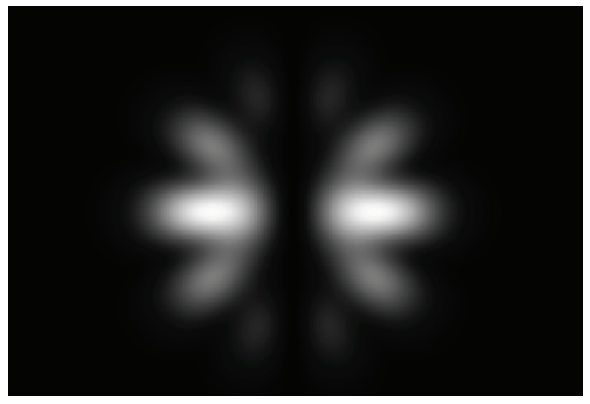

b)

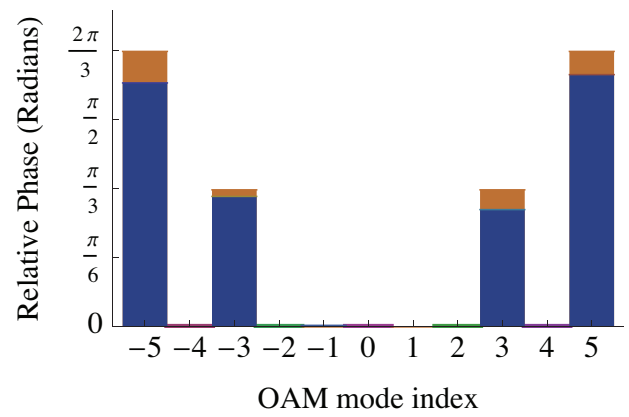

Figure 3: Simulation showing (a) the input intensity distribution and (b) the measured relative phase between component modes of an OAM superposition as measured by our direct measurement method (orange is expected, blue is measured).

\section{References}

[1] J. S. Lundeen, B. Sutherland, A. Patel, C. Stewart, and C. Bamber, "Direct measurement of the quantum wavefunction," Nature 474(7350), 188 (2011).

[2] G. C. G. Berkhout, M. P. J. Lavery, J. Courtial, M. W. Beijersbergen, and M. J. Padgett, "Efficient 
Sorting of Orbital Angular Momentum States of Light," Physical Review Letters 105(15), 153,601 (2010).

[3] I. Moreno, J. L. Martinez, and J. A. Davis, "Two-dimensional polarization rotator using a twisted-nematic liquid-crystal display," Applied Optics 46(6), 881-887 (2007). 